\title{
IMMERSIONS OF COMPLEX HYPERSURFACES $\left({ }^{1}\right)$
}

\author{
BY
}

\author{
STANLEY R. SAMSKY(2)
}

\begin{abstract}
The varieties $V^{n}(d)=\left\{\left[z, \ldots, z_{n}\right] \in C P^{n}: z_{0}^{d}+\cdots+z_{n}^{d}=0\right.$, $d>0\}$ form a class of manifolds containing the complex projective spaces. Maps from $V^{n}(d)$ to $V^{k}(e)$ are partially characterized by a "degree". We prove some nonimmersion results which are phrased in terms of this degree, and which generalize the results of S. Feder [4] on complex projective spaces.
\end{abstract}

Introduction. Given two manifolds (smooth, oriented, without boundary) $M$ and $N$, and a map $f: M \rightarrow N$, one would like to decide whether the homotopy class $[f]$ in $[M, N]$ contains an immersion or an embedding. The bulk of research has dealt with the case $N=R^{n}$ (see, e.g., I. James [7] for a general summary when $M$ is a projective space). S. Feder [4] dealt with this problem in the case where both $M$ and $N$ were complex projective spaces, and K. H. Mayer [8] has studied the case where $N$ is a complex projective space, and $M$ is in a large class of manifolds.

In this paper, we generalize the work of Feder to the case where $M$ and $N$ are certain projective algebraic varieties, $V^{n}(d)$, which represent multiples of the generator of $H_{2 n-2}\left(C P^{n} ; Z\right)$. Maps $V^{n}(d) \stackrel{f}{\rightarrow} V^{k}(e)$ are partially characterized by a degree, and we formulate the following basic question in terms of this degree.

Suppose $f: V^{n}(d) \rightarrow V^{k}(e)$ has $\operatorname{deg} f=\beta$. Can $f$ be homotopic to an immersion?(3)

We show that if $k$ is large enough compared with $n$, there are embeddings of $V^{n}(d)$ in $V^{k}(e)$ with any degree.

Later, we use classical methods to get at complex analytic immersions, proving, among others,

Received by the editors April 20, 1973 and, in revised form, July 23, 1974.

AMS (MOS) subject classifications (1970). Primary 57D40; Secondary 55B15, 55F 20, 57D20.

Key words and phrases. Embeddings, immersions, $K$ theory, normal bundles, characteristic classes, hypersurfaces.

(1) Revision of the author's Ph.D. Thesis at the University of California, Berkeley, 1972.

(2) Partially supported by the NSF and NRC during the preparation of this paper.

(3) To avoid trivial cases $\left(V^{1}(d)\right.$ is a finite set of points) we will assume that $n \geq 2$. 
Theorem. Let $f: V^{n}(d) \rightarrow C P^{k}$ be an analytic immersion, with $k \leq$ $2 n-3$. Then $\operatorname{deg} f=1$.

In \$III, we use the techniques of K-theory (see Feder [4]) to prove other nonimmersion results.

The author would like to thank Dennis Barden, Alan Durfee, Arnold Kas, and especially his advisor, Emery Thomas, for many valuable discussions on the subject of this paper.

I. The varieties $V^{n}(d)$.

1. Preliminaries. R. Thom [10] proved that for any oriented $n$-manifold $M$ every integral homology class $\gamma \in H_{n-2}(M)$ can be represented by an embedded submanifold $N_{\gamma}$; i.e., if $i: N_{\gamma} \rightarrow M$ is the embedding, then $i_{*}\left[N_{\gamma}\right]=$ $\gamma$. If $M$ is a complex projective space, $M=C P^{n}$, the manifolds $N_{\gamma}$ are particularly simple, and we recall a few facts without proof:

Proposition (1). Let $V^{n}(d)=\left\{\left[z_{0}, z_{1}, \ldots, z_{n}\right] \in C P^{n} \mid z_{0}^{d}+\cdots+z_{n}^{d}=0\right.$, $d>0, d \in Z\}$. Then $V^{n}(d)$ is a nonsingular complex $(n-1)$-dimensional sub. variety of $C P^{n}$, and hence a complex $(n-1)$ manifold. Considered as a homology class, $V^{n}(d)$ represents $d$ times the generator $\left[C P^{n-1}\right]$ of $H_{2 n-2}\left(C P^{n}\right)$.

If $y \in H^{2}\left(C P^{n}\right)$ is the generator then $V^{n}(d)$ is the Poincare dual to $d y$. F. Hirzebruch has shown [5] that the normal bundle of $V^{n}(d)$ in $C P^{n}$ is given by $\nu\left(V^{n}(d)\right)=i^{\sharp}\left(H_{n}^{d}\right)$, where $H_{n}$ is the Hopf bundle over $C P^{n}$, and $i^{\#}$ is the pullback induced by the inclusion $i$. (This is true because a line bundle is classified by its first Chern class.)

We will write $\zeta_{n}=i^{\#}\left(H_{n}\right)$, so $\zeta_{n}^{d}=i^{\#}\left(H_{n}^{d}\right)$. We will often use the fact

$$
\nu\left(V^{n}(d)\right)=\zeta_{n}^{d}
$$

2. The cohomology of $V^{n}(d)$. We will compute as much of the cohomology of $V^{n}(d)$ as we need:

Proposition (2). Let $i: V^{n}(d) \rightarrow C P^{n}$ be the canonical inclusion. Then

(i) $i^{*}: H^{k}\left(C P^{n}\right) \rightarrow H^{k}\left(V^{n}(d)\right)$ is an isomorphism if $k<n-1$ (similarly in homology) and if $k \neq n-1, H^{k}\left(C P^{n-1}\right) \cong H^{k}\left(V^{n}(d)\right)$;

(ii) $H^{n-1}\left(V^{n}(d)\right)$ is a free abelian group and

$$
\operatorname{rank}\left(H^{n-1}\left(V^{n}(d)\right)\right)= \begin{cases}\frac{(d-1)^{n+1}+2 d-1}{d} & \text { (noodd), } \\ \frac{(d-1)^{n+1}-d+1}{d} & \text { (neven); }\end{cases}
$$

(iii) if $y_{n}=i^{*} y$, then $y_{n}$ generates a polynomial subalgebra of $H^{*}\left(V^{n}(d)\right)$ and $y_{n}^{k}=d \cdot$ generator of $H^{2 k}\left(V^{n}(d)\right)$, if $2 k \geq n-1$. 
Proof (Sketch). (i) This is essentially the Lefshetz theorem on hyperplane sections. (See A. Andreotti and T. Frankel [2].)

(ii) Freeness is an application of duality and the universal coefficient theorem. The rank of $H^{n-1}\left(V^{n}(d)\right)$ can be computed by observing that the Euler class $e\left(V^{n}(d)\right)=c_{n-1}\left(V^{n}(d)\right)$ and computing $c_{n-1}$ from Lemma (3) (below).

Lemma (3). Stably, $r\left(V^{n}(d)\right)=(n+1) \zeta_{n}-\zeta_{n}^{d}$.

Proof. An immediate consequence of $(*)$.

(iii) This follows from the fact that $i_{*}\left[V^{n}(d)\right]=d \cdot$ generator. We are now in a position to make the main definition.

Definition. Let $f: V^{n}(d) \rightarrow V^{k}(e)$ be a continuous map. The degree of $f, \operatorname{deg}(f)$, is the unique integer $\beta$ for which $f^{*} y_{k}=\beta y_{n}$, where $y_{m} \in H^{2}\left(V^{m}(a)\right)$ is the generator. $\left({ }^{4}\right)$

Note. If $e=1$, this agrees with the definition in Feder [4], and in this case two maps are homotopic iff they have the same degree.

3. A positive result. In $\S \S \mathrm{II}$ and III, we will prove a number of negative results. If these results are to have any real content, it behooves us to show that in certain cases embeddings and immersions do exist.

Theorem (4). For any integer $\beta>0$, there exist an analytic embedding $g: V^{n}(d) \rightarrow V^{2 n}(e)$ and an analytic immersion $h: V^{n}(d) \rightarrow V^{2 n-1}(e)$, of degree $\beta$, for all $d, e>0$.

(See Feder [4] for the case $d=e=1$.)

(1) The standard inclusion evidently has degree 1.

(2) The Veronese embedding: One can construct [5] an embedding $C P^{n}$ $\rightarrow C P^{N}, N=\left(\begin{array}{c}n+\beta \\ \beta\end{array}\right)-1$, which is analytic and has the required degree $\beta>0$. One merely sends $\left[z_{0}, \ldots, z_{n}\right]$ to $\left[P_{1}, \ldots, P_{N}\right]$, where the $P_{i}$ are all monomials of total degree $d$.

(3) We now construct an embedding $\zeta: C P^{N} \rightarrow V^{2 N+1}(e)$ with degree 1 .

We can think of $V^{2 N+1}(e)$ as the set of $\left[z_{0}, \ldots, z_{2 N+1}\right]$ with $\left(z_{0}^{e}+z_{1}^{e}\right)$ $+\left(z_{2}^{e}+z_{3}^{e}\right)+\cdots+\left(z_{2 N}^{e}+z_{2 N+1}^{e}\right)=0$. Let $\omega$ be a fixed eth of -1 . Then $\zeta\left[z_{0}, \ldots, z_{N}\right]=\left[z_{0}, \omega z_{0}, \ldots, z_{N}, \omega z_{N}\right] \in C P^{2 N+1}$. Note that $\operatorname{Im}(\zeta) \in V^{2 N+1}(e)$, because $z_{0}^{d}+\left(\omega z_{0}\right)^{d}+\cdots+z_{N}^{d}+\left(\omega z_{N}\right)^{d}=0$. $\zeta$ is obviously an algebraic embedding, and a calculation like that in [5] shows $\operatorname{deg}(\zeta)=1$.

(4) If we put (1), (2) and (3) together, we have for any $\beta>0, k \geq 2\left(\begin{array}{c}n+\beta \\ \beta\end{array}\right)$ -1 , an analytic embedding $V^{n}(d) \stackrel{\rightarrow}{\rightarrow} V^{k}(e)$. An argument using Sard's theorem gives the smaller values for $k$.

(4) One can extend this in the obvious way to any two complex projective hypersurfaces. 
To get a $C^{\infty}$ embedding of degree 0 , note that $V^{n}(d)$ embeds in $R^{4 n-4}$ and hence in $V^{2 n-1}(e)$ with degree $0 .(5)$ If we are willing to take the embeddings and immersions to be $C^{\infty}$, we can get all degrees. This follows from part (2) of the proof of Theorem (4) and the fact that complex conjugation (which is not analytic) mapping $C P^{k} \rightarrow C P^{k}$ is an automorphism of degree -1 .

Notes. (1) Feder [4] proves a version of this for $d=e=1$ in which dimensional restrictions are a bit better. (2) It was pointed out to me by Emery Thomas that in any homotopy class of maps from a $2 n$ manifold to a simply connected $4 n$ manifold, one can find an embedding which gives a nicer $C^{\infty}$ existence result.

II. Computations from ordinary cohomology-characteristic class arguments. In this section, we prove a simple necessary condition for the existence of maps of degree $\beta$ from $V^{n}(d)$ to $V^{k}(e)$, and we rule out the existence of analytic immersions in some cases.

1. Two general results.

Proposition (1). Let $\beta$ be an integer. If $e$ does not divide $\beta^{n-1} d$ then there exists no continuous map $f: V^{n}(d) \rightarrow V^{k}(e)$ with $\operatorname{deg} f=\beta, k<2 n-1$.

Proof. For any $f, \operatorname{deg} f=\beta$, we compute $\left\langle y_{k}^{n-1}, f_{*}\left[V^{n}(d)\right]\right\rangle$ in $V^{k}(e)$ and in $V^{n}(d)$. The result follows by comparing the answers:

$$
\left\langle y_{k}^{n-1}, f_{*}\left[V^{n}(d)\right]\right\rangle=\left\langle f^{*} y_{k}^{n-1},\left[V^{n}(d)\right]\right\rangle=\left\langle\beta^{n-1} y_{n}^{n-1},\left[V^{n}(d)\right]\right\rangle=\beta^{n-1} d .
$$

On the other hand, suppose $k<2 n-1$; then for some integer $\gamma, f_{*}\left[V^{n}(d)\right]=$ $\gamma x_{2 n-2}$, where $x_{2 n-2}$ is the generator of $H_{2 n-2}\left(V^{k}(e)\right)=Z$. Hence

$$
\left\langle y_{k}^{n-1}, f_{*}\left[V^{n}(d)\right]\right\rangle=\left\langle y_{k}^{n-1}, \gamma x_{2 n-2}\right\rangle=\gamma e
$$

(since $2 n-1>k$ ) so $\gamma e=\beta^{n-1} d$, giving the proposition.

Note. If $k>2 n-1$, we get $\gamma=\beta^{n-1} d$, i.e. no information, while if $k=$ $2 n-1, H_{2 n-2}\left(V^{k}(e)\right)$ has too many generators to apply this analysis.

Corollary (2). Suppose $e$ and $d$ are relatively prime. Then if $f: V^{n}(d)$ $\rightarrow V^{k}(e)$ is a continuous map $(k<2 n-1),(\operatorname{deg} f)^{n-1}$ is divisible by $e$. In particular, if $e$ is prime, $\operatorname{deg} f$ is a multiple of $e$.

Corollary (3). Let $k<2 n-1$. If there exists a map $f: V^{n}(d) \rightarrow V^{k}(e)$ with $(\operatorname{deg} f, e)=1$, then $e$ divides $d$.

Proposition (4). Let $f: V \rightarrow W$ be a nonconstant holomorphic map be-

(5) A holomorphic immersion of degree $\mathbf{0}$ is impossible (see II, Corollary (5)). 
tween nonsingular complex projective hypersurfaces. Then $\operatorname{deg} f>0$.

Proof (Sketch). Observe that a holomorphic map between nonsingular projective varieties is algebraic (since its graph is a projective variety and hence, by Chow's theorem, is algebraic). But an algebraic map is just the restriction of a map polynomial in each coordinate and thus (by the argument mentioned in Theorem (4), $\$ \mathrm{I}$ ) has degree $>0$ if it is nonconstant.

Corollary (5). If $f$ is a holomorphic immersion of $V^{n}(d)$ in $V^{k}(e)$, then $\operatorname{deg} f>0$.

Proof. By Proposition (4), if $\operatorname{deg} f=0, f$ is a constant map and hence cannot be an immersion.

2. Arguments involving Chern classes. If $f: M^{n} \rightarrow N^{n+k}$ is an analytic immersion of complex manifolds, it is well known that for the stable normal bundle, $\nu_{f}=f^{\#} r_{N}-\tau_{M}$, the $(k+1)$ st Chern class, $c_{k+1}\left(\nu_{f}\right)=0$. We compute the total Chern class of an analytic immersion $V^{n}(d) \stackrel{f}{\rightarrow} V^{k}(e)$ and apply the observation above.

Lemma (6). Suppose $f: V^{n}(d) \rightarrow V^{k}(e)$ is a continuous map with $\operatorname{deg} f$ $=\beta$. Then the stable normal bundle of $f$ is given by

$$
\nu_{f}=(k+1) \zeta_{n}^{\beta}-\gamma_{n}^{\beta}-(n+1) \zeta_{n}+\zeta_{n}^{d}
$$

and the total Chern class of $\nu$, is

$$
c\left(\nu_{f}\right)=\left(1+\beta y_{n}\right)^{-1}\left(1+e \beta y_{n}\right)^{-1}\left(1+y_{n}\right)^{-n-1}\left(1+d y_{n}\right) .
$$

Proof. Recall that $r\left(V^{n}(d)\right)=(n+1) \zeta_{n}-\zeta_{r}^{d}$ (stably). Since $\zeta_{n}$ is a line bundle, it is classified by its first Chern class $c\left(\zeta_{n}\right)=1+y_{n}$, and $f^{\sharp}\left(\zeta_{n}\right)$ is a line bundle with

$$
c\left(f^{H}\left(\zeta_{n}\right)\right)=f^{*} c\left(\zeta_{n}\right)=f^{*}\left(1+y_{n}\right)=1+\beta y_{n} .
$$

This clearly implies $f^{\#}\left(\zeta_{n}\right)=\zeta_{n}^{\beta}$.

The first part of the lemma now follows by a computation, and the second part by the product formula for Chern classes.

The plan of attack is now to compute $c_{k-n+1}\left(\nu_{f}\right)$ for an analytic immersion $f: V^{n}(d) \rightarrow V^{k}(e)$. We first present three algebraic lemmas. (The author would like to thank D. Barden for pointing out a simpler proof of this lemma.)

Lemma (7). (A) The coefficient of $y^{n}$ in $(1+\beta y)^{m+n} /(1+y)^{n+1}$ is $\left(\begin{array}{c}m+n \\ n\end{array}\right)(\beta-1)^{m}$.

(B) The coefficient of $y^{m}$ in $(1+\beta y)^{2 m}(1+d y) /(1+y)^{m+2}$ is 


$$
\left(\begin{array}{c}
2 m+1 \\
m+1
\end{array}\right)(\beta-1)^{m}+(d-\beta)\left(\begin{array}{c}
2 m \\
m+1
\end{array}\right)(\beta-1)^{m-1}
$$

(C) The coefficient of $y^{m}$ in $\left(1+d_{1} y\right) \cdots\left(1+d_{m} y\right) /(1+e y)$ is $\Pi_{i=1}^{m}\left(d_{i}-e\right)$.

Proof. We do only (A). The others are similar.

Let us call $c_{m}(\beta)$ the coefficient of $y^{m}$. If we differentiate with respect to $\beta$ and equate coefficients of $y^{m}$, we get

$$
(m+n) c_{m-1}(\beta)=c_{m}^{\prime}(\beta) .
$$

Note that for all $m, n$, we get a polynomial of degree $m-1$ if $\beta=1$, so $c_{m}(1)=0$. Hence $(\beta-1)$ divides $c_{m}(\beta)$ for all $m \geq 1$, and by $(*)$ we see that $(\beta-1)$ is an $m$-fold factor. We get $c_{m}(\beta)=\left(\begin{array}{c}m_{m}^{+n} \\ m\end{array}\right)(\beta-1)^{m}$ by checking the coefficient of $\beta^{m}$.

Theorem (8). For $k \leq 2 n-2$, an analytic immersion $f: V^{n}(d) \rightarrow V^{k}(e)$ has $\operatorname{deg} f=1$ if and only if $d=e$ or $e=1$.

Proof. Notice first that it suffices to look at the single case $k=2 n-2$, for we have a degree 1 embedding of $V^{l}(e)$ in $V^{l+1}(e)$ gotten by considering $V^{l}(e)$ in $C P^{l+1}$ as $V^{l+1}(e)$ intersected with the hyperplane $\left\{z: z_{l+1}=0\right\}$. We notice that under the hypothesis $\beta=1$,

$$
c\left(\nu_{f}\right)=\left(1+y_{n}\right)^{n-2}\left(1+e y_{n}\right)^{-1}\left(1+d y_{n}\right) .
$$

Now $\nu_{f}$ is a complex $(n-2)$-plane bundle, so $c_{n-1}(f) y_{n}^{n-1} \equiv c_{n-1}\left(\nu_{f}\right)=0$.

By Lemma $(7)(C), c_{n-1}(f)=(1-e)^{n-2}(d-e)$. Hence $d=e$ or $e=1$. The converse is obvious.

Note. If $k>2 n-2$, we have already shown that immersions of arbitrary positive degree exist, so the theorem cannot be improved.

Theorem (9). Let $f: V^{n}(d) \rightarrow V^{k}(1)=C P^{k-1}$ be an analytic immersion, where $k \leq 2 n-2$. Then $\operatorname{deg} f=1$.

Proof. It suffices as usual to look only at $k=2 n-2$. In this case, $c_{n-1}(f)=0$, and by (2)

$$
c(\nu(f))=\left(1+\beta y_{n}\right)^{2 n-2}\left(1+d y_{n}\right)\left(1+y_{n}\right)^{-n-1} .
$$

We apply (7)(B), to get

$$
c_{n-1}(f)=\left(\begin{array}{c}
2 n-1 \\
n
\end{array}\right)(\beta-1)^{n-1}+(d-\beta)\left(\begin{array}{c}
2 n-2 \\
n
\end{array}\right)(\beta-1)^{n-2},
$$


so we see, after some arithmetic,

$$
n(\beta-1)^{n-2}[\beta-(2-d+(d-1) / n)]=0 .
$$

So we get either $\beta=1$ or $\beta=2-d+(d-1) / n$ which can never be an integer $>1$ by Corollary (5).

Theorem (10). Suppose $f: V^{n}(d) \rightarrow V^{k}(e)$ is an analytic immersion, $k \leq$ $2 n-2$ and $\operatorname{deg} f=d / e$. Then $d=e$ and $\operatorname{deg} f=1$.

Proof. As usual it suffices to look at $k=2 n-2$. If $\beta=d / e$, we see that

$$
c\left(\nu_{f}\right)=\left(1+\beta y_{n}\right)^{2 n-1} /\left(1+y_{n}\right)^{n+1},
$$

and by Lemma (5)(A)

$$
c_{n-1}(f)=\left(\begin{array}{c}
2 n-1 \\
n
\end{array}\right)(\beta-1)^{n-1}=0
$$

so $\beta=1$. It has proved very difficult, in general, to solve the equation $c_{n-1}(f)=0$ for $\beta$. However, one has the following general result:

Theorem (11). If $k \leq 2 n-2$, the set $\{\operatorname{deg}(f): f$ an analytic immersion of $V^{n}(d)$ in $\left.V^{e}(k)\right\}$ has at most $(k-n+1)$ elements.

Proof. If we expand (2) and write down the coefficient of $y_{n}^{k-n+1}$, we get a polynomial of degree $(k-n+1)$ in $\beta$. Finally, if $n$ is fairly small, one can get explicit results, for instance:

Theorem (12). An analytic immersion of $V^{3}(d)$ in $V^{4}(d)$ must have degree 1.

III. $K$-theory techniques. In this section, $K$-theory characteristic class arguments will be used to prove a nonimmersion result for the smooth case. We follow Feder [4] closely.

1. Definitions. We assume known the definition and elementary properties of $K U(X)$ and $K O(X)$. See D. Husemoller [6] for the relevant material.

Recall that the exterior power operation on vector spaces extends to the usual operation $\lambda^{i}$ on $K(X)$ (here $K(X)$ will be either $K U(X)$ or $K O(X)$ ). The $\lambda^{i}$ have the following properties $\xi, \eta \in K(X)$,

(i) $\lambda^{0}(\xi)=1$,

(ii) $\lambda^{1}(\xi)=\xi$,

(iii) $\lambda^{r}(\xi \oplus \eta)=\Sigma_{i+j=r} \lambda^{i}(\xi) \lambda^{j}(\eta)$.

Definition. Let $\xi$ be a real $k$-plane bundle. We say $\xi$ is a spin bundle if $w_{1}(\xi)+w_{2}(\xi)=0$ 
Proposition (1). (See J. Milnor [0], D. Husemoller [6].) If $\xi$ is a real $k$-dimensional spin bundle, then $\xi$ admits a spin representation, i.e. there is a complex self-conjugate bundle $\Delta(\xi)$, such that

$$
a_{k} \Delta(\xi) \cdot \Delta(\xi)=\lambda^{0}\left(\xi^{1}\right)+\lambda^{1}\left(\xi^{1}\right)+\cdots+\lambda^{k}\left(\xi^{1}\right)
$$

where $\xi^{1}=\xi \otimes C$, and

$$
a_{k}= \begin{cases}1, & k \equiv 0(\bmod 2) \\ 2, & k \equiv 1(\bmod 2)\end{cases}
$$

Definition. We do the standard trick to make the $\lambda^{i}$ 's multiplicative, and define

$$
\lambda_{t}(\xi)=\sum_{i=0}^{\infty} \lambda^{i}(\xi) t^{i} \in K(X)[t] \quad \text { for } \xi \in K(X) .
$$

Note. (a) $\lambda_{t}(\xi+\eta)=\lambda_{y}(\xi) \lambda_{t}(\eta)$,

(b) $\lambda_{t}(a)=1+t a$, if $a$ is a line bundle.

Proposition (2). Suppose $\xi$ is a real spin bundle over $X$. Then there exists a self-conjugate element $Z \in K U(X)$ such that

$$
A_{k} Z^{2}=\lambda_{1}(\xi \otimes C) \text { where } A_{k}= \begin{cases}1, & k \equiv 0(\bmod 2), \\ 2, & k \equiv 1(\bmod 2) .\end{cases}
$$

Proof. Put (1) and (2) together. $Z=\Delta(\xi)$.

2. Smooth immersions. We gather together a few general results arising from $\$ I$ which will be applied in $\$ I I I$ to the case of the $V^{n}(d)$.

Let $M$ and $N$ be smooth, compact manifolds without boundary of dimensions $n$ and $n+k$, respectively. We denote by $r(M), r(N)$ their tangent bundles and also (by abuse of notation) their equivalence classes in $K O(M)$, $\mathrm{KO}(\mathrm{N})$.

Definition. For any map $f: M \rightarrow N$, we define the stable normal bundle of $f, \nu(f)$, by

$$
\nu(f)=f^{\#} r(N)-f(M) \in K O(M) .
$$

In general, $\nu(f)$ depends only on the homotopy class $[f] \epsilon[M, N]$.

Theorem (3). If $[f] \in[M, N]$ contains an immersion, and if $\nu(f)$ is a spin bundle, then there is a self-conjugate $Z \in K U(M)$ with $a_{k} Z^{2}=\lambda_{1}(\nu(f) \otimes C)$.

Proof. Apply Proposition (1), since $\nu(f)$ is a k-plane bundle. 
3. The K-theory of $V^{n}(d)$ and the nonimmersion theorem. Our aim is to compute a sufficiently large part of $K U\left(V^{n}(d)\right)$ to allow us to apply Theorem (3). Using the Chern character, we compute $K U\left(V^{n}(d)\right) \otimes Q$, and a pleasant property of polynomials allows us to see what the self-conjugate $Z$ of Theorem (3) looks like based on our knowledge of what the self-conjugates in $K U\left(C P^{n}\right)$ are.

We recall (see E. Dyer [3]) that there exists a ring homomorphism $c h$ : $K U(X) \rightarrow H^{\text {even }}(X ; Q)$, which is a rational isomorphism. A straightforward consequence of this is

Theorem (4). Suppose $n$ is even. Then the map $i^{\#}: K U\left(C P^{n}\right) \otimes Q \rightarrow$ $K U\left(V^{n}(d)\right) \otimes Q$ induced by inclusion is surjective, and its kernel is the ideal generated by $h_{n}^{n}$, where $h_{n}=H_{n}-1$ is the reduced Hopf bundle over $C P^{n}$.

Proof. We note that for $n$ even,

$$
i^{*}: H^{\text {even }}\left(C P^{n} ; Q\right) \rightarrow H^{\text {even }}\left(V^{n}(d) ; Q\right)
$$

is an epimorphism, and $\operatorname{ker} i^{*}$ is generated by $y^{n}$, where $y$ is the image of the generator of $H^{2}\left(C P^{n}\right)$ in $H^{2}\left(C P^{n} ; Q\right)$. (The generator of $H^{2 k}\left(C P^{n}\right), k<$ $n$, maps to an integral multiple of the generator of $H^{2 k}\left(V^{n}(d)\right)$.) We have the commutative diagram:

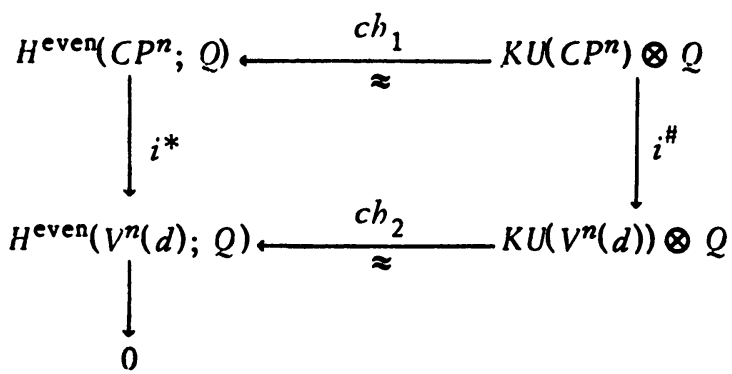

This clearly implies $i^{\#}$ is surjective and that $\operatorname{ker} i^{\#}=\operatorname{ker}\left(c h_{2}^{-1} i^{*} c h_{1}\right)$. But

$$
\begin{aligned}
\operatorname{ker}\left(c h_{2}^{-1} i^{*} c h_{1}\right) & =c h_{1}^{-1} \operatorname{ker} i^{*} \quad \text { (the } c h_{i} \text { are isomorphisms) } \\
& =\operatorname{ch}_{1}^{-1}\left(y^{n}\right) .
\end{aligned}
$$

For the Hopf bundle $H_{n}, c_{1}\left(H_{n}\right)=y$, so $c h_{1}\left(H_{n}-1\right)=c h_{1}\left(H_{n}\right)-1=e^{y}-1$ thus,

$$
\operatorname{ch}_{1}\left(H_{n}-1\right)^{n}=\left(e^{y}-1\right)^{n}=\left(y+y^{2} / 2 !+\cdots+y^{n} / n !\right)^{n}=y^{n},
$$

i.e. $\operatorname{ch}_{1}^{-1} y^{n}=\left(H_{n}-1\right)^{n}=h_{n}^{n}$.

Corollary (5). $K U\left(V^{n}(d)\right) \otimes Q=Q\left[\eta_{n}\right] /\left(\eta_{n}^{n}\right)$, where $\eta_{n}=i^{\#} h_{n}$. 
Proof (J. F. Adams [1]). $K U\left(C P^{n}\right)=Z\left[h_{n}\right] /\left(h_{n}^{n+1}\right)$.

We are interested in the algebra of self-conjugate elements of $K U\left(V^{n}(d)\right)$. It is evident (since conjugation commutes with maps) that a self-conjugate element of $K U\left(V^{n}(d)\right) \otimes Q$ is the image of a self-conjugate element of $K U\left(C P^{n}\right) \otimes Q$. We have the following characterization of self-conjugate elements of $K U\left(C P^{n}\right)$ (see Feder [4]): Let $\bar{h}_{n}=\bar{H}_{n}-1$. Since $H_{n} \bar{H}_{n}=1$ in $K U\left(C P^{n}\right)$ we have

$$
\bar{h}_{n}+h_{n}+\bar{h}_{n}+h_{n}=0 \text { and } \bar{h}_{n}=-h_{n} /\left(1+h_{n}\right)=-h_{n}\left(1-h_{n}+h_{n}^{2}-\cdots \pm h_{n}^{n}\right) \text {. }
$$

Set $z=h_{n}+\bar{h}_{n}$. Then $h_{n} \bar{h}_{n}=-z$, and since, on $C P^{n}$, self-conjugate bundles are obviously symmetric in $h_{n}$ and $\bar{h}_{n}$, we get

Lemma (6). Any self-conjugate element of $K U\left(C P^{n}\right)$ is an integral polynomial in $z$.

Note. If $n$ is even, $z^{n / 2}=h_{n}^{n}$.

Corollary (7). If $n$ is even, every self-conjugate element of $K U\left(V^{n}(d)\right)$ is a rational polynomial in $X=i^{\#} z$; indeed the self-conjugates of $K U\left(V^{n}(d)\right.$ ) $\otimes Q$ form a truncated polynomial algebra $\left.Q[X] /\left(X^{[n / 2}\right]\right)$.

Proof. The second part of the corollary is obvious by the remark preceding Corollary (7). According to Dyer [3], since $H^{*}\left(V^{n}(d)\right)$ is torsion free, $K U\left(V^{n}(d)\right)$ is monomorphically embedded in $K U\left(V^{n}(d)\right) \otimes Q$, which implies the first part of the corollary.

We now have sufficient data on hand to apply Theorem (3). First we slightly strengthen Theorem (3) in our special case,

Theorem ( $\left.3^{\prime}\right)$. Let $\{f\} \in\left[V^{n}(d), V^{k}(e)\right]$ be the set of maps $g$ such that $\operatorname{deg}(g)=\operatorname{deg}(f)$. Then, if $\{f\}$ contains an immersion, and $\nu(f)$ is a spin bundle, there is a self-conjugate $z \in K U\left(V^{n}(d)\right)$ with $z^{2}=\lambda_{1}(\nu(f) \otimes C)$.

Proof. According to Lemma (6) of $\$ I I$,

$$
\nu(f)=(k+1) \zeta_{n}^{\beta}-\zeta_{n}^{\beta}-(n+1) \zeta_{n}+\zeta_{n}^{d},
$$

where $\beta=\operatorname{deg}(f)$, so $\nu(f)$ depends only on $\operatorname{deg}(f)$. Since $\operatorname{dim}_{R} \nu(f)=2 k-2 n$, we get $\lambda_{1}(\nu(f) \otimes C)=A_{2 k-2 n} z^{2}=z^{2}$. ( $(\nu(f)$ denotes the real bundle underlying $\nu(f)$.) We are now able to compute $\lambda_{t}(\nu(f) \otimes C)$ in standard fashion (see Feder [4]).

Definition. Let $S_{d}\left(X_{1}, X_{2}\right)$ be the Newton polynomial defined by $t_{1}^{d}+$ $t_{2}^{d}=S_{d}\left(t_{1}+t_{2}, t_{1} t_{2}\right)$ and set

$$
T_{d}(X)=S_{d}(X+2,1)-2 .
$$


Note. (i) $T_{1}(X)=X, T_{2}(X)=4 X+X^{2}, T_{3}(X)=9 X+6 X^{2}+X^{3}$.

(ii) The constant term of $T_{d}(X)$ is zero (if $x=0, s_{d}(X+2,1)=2$ ).

(iii) $T_{d}(X)=d^{2} X+$ higher order terms (see Feder [4]). Recall, if $\rho$ is a complex bundle, $\rho \otimes C=\rho \oplus \bar{\rho}$; hence $\xi=\nu(f) \otimes C=\nu(f)$ $\oplus \overline{\nu(f)}$, and by (5) we have

(7) $\quad \xi=(k+1)\left(\zeta_{n}^{\beta}+\bar{\zeta}_{n}^{\beta}\right)-\left(\zeta_{n}^{\beta}+\bar{\zeta}_{n}^{\beta}\right)-(n+1)\left(\zeta_{n}+\bar{\zeta}_{n}\right)+\left(\zeta_{n}^{d}+\bar{\zeta}_{n}^{d}\right)$.

Now, $\zeta_{n}^{r}$ is a line bundle, for any positive integer $r$, so $\lambda_{t}\left(\zeta_{n}^{r}\right)=1+t \zeta_{n^{0}}^{r}$ Thus,

$$
\begin{aligned}
\lambda_{t}(\xi)= & {\left[\left(1+t \zeta_{n}\right)\left(1+t \bar{\zeta}_{n}^{\beta}\right)\right]^{k+1} \cdot\left[\left(1+t \zeta_{n}^{e \beta}\right)\left(1+t \bar{\zeta}_{n}^{e \beta}\right)\right]^{-1} } \\
& \cdot\left[\left(1+t \zeta_{n}\right)\left(1+t \bar{\zeta}_{n}\right)\right]^{-(n+1)} \cdot\left[\left(1+t \zeta_{n}^{d}\right)\left(1+t \bar{\zeta}_{n}^{d}\right)\right]
\end{aligned}
$$

But $\zeta_{n} \bar{\zeta}_{n}=1$, so we can rewrite (8) as

$$
\begin{aligned}
\lambda_{t}(\xi)= & {\left[1+t\left(\zeta_{n}^{\beta}+\bar{\zeta}_{n}^{\beta}\right)+t^{2}\right]^{k+1} \cdot\left[1+t\left(\zeta_{n}^{\beta}+\bar{\zeta}_{n}^{e}\right)+t^{2}\right]^{-1} } \\
& \cdot\left[1+t\left(\zeta_{n}+\bar{\zeta}_{n}\right)+t^{2}\right]^{-(n+1)} \cdot\left[1+t\left(\zeta_{n}^{d}+\bar{\zeta}_{n}^{d}\right)+t^{2}\right]
\end{aligned}
$$

Finally, we notice that

$$
\zeta_{n}^{-k}+\bar{\zeta}_{n}^{-k}=\zeta_{n}^{k}+\bar{\zeta}_{n}^{k}=S_{k}\left(\zeta_{n}+\bar{\zeta}_{n}, \zeta_{n} \bar{\zeta}_{n}\right)=S_{k}(X+2,1)=T_{k}(X)+2
$$

Hence, we have proved

Lemma (8). If $f: V^{n}(d) \rightarrow V^{k}(e)$ has degree $\beta$, then

$$
\begin{aligned}
\lambda_{t}(\nu(f) \otimes C)= & {\left[1+t\left(T_{\beta}(X)+2\right)+t^{2}\right]^{k+1} \cdot\left[1+t\left(T_{e \beta}(X)+2\right)+t^{2}\right]^{-1} } \\
& \cdot\left[1+t(X+2)+t^{2}\right]^{-(n+1)} \cdot\left[1+t\left(T_{d}(X)+2\right)+t^{2}\right]
\end{aligned}
$$

We are aiming towards applying Theorem (3), so we must make sure that $\nu(f)$ is a spin bundle. Since $H^{1}\left(V^{n}(d) ; Z_{2}\right)=0, w_{1}(\nu(f))=0$, so we need only $w_{2}(\nu(f))=0$.

Lemma (9). If $f: V^{n}(d) \rightarrow V^{k}(d)$ has degree $\beta$, then $\nu(f)$ is a spin bundle iff $\beta(k+e+1)+d \equiv n+1(\bmod 2)$.

Proof. If $w_{1}=0, w_{2}$ is additive, so by (5),

$$
w_{2}(\nu(f))=[(k+1) \beta-e \beta-(n+1)+d] \rho_{2} y,
$$

where $\rho_{2}$ is reduction $\bmod 2$.

Proposition (10). Suppose $n$ is even, and there exists an immersion f: 
$V^{n}(d) \rightarrow V^{k}(e)$ satsifying

(a) $\operatorname{deg}(f)=\beta$,

(b) $\beta(k+e+1)+d \equiv 1(\bmod 2)$;

then

$2^{k-n}[1+1 / 4 T(X)]^{(k+1) / 2}[1+1 / 4 X]^{-(n+1) / 2}\left[1+1 / 4 T_{d}(X)\right]^{1 / 2}\left[1+1 / 4 T_{e \beta}(X)\right]^{-1 / 2}$

is an integral polynomial in $X$.

Proof.

$\lambda_{1}(\nu(f) \otimes C)=\left[4+T_{\beta}(X)\right]^{k+1}[4+X]^{-(n+1)}\left[4+T_{d}(X)\right]\left[4+T_{c \beta}(X)\right]^{-1}$

and (b) says that $\nu(f)$ is a spin bundle. Hence, by Theorem $\left(3^{\prime}\right), \lambda_{1}(\nu(f) \otimes C)$ $=z^{2}$, where $z$ is self-conjugate. By Corollary (7), we know $z$ is a rational polynomial in $X$, but polynomials have the pleasant property:

If $p \in Z[X]$ and $q \in Q[X]$ and $p=q^{2}$, then $q \in Z[X]$. This says $\lambda_{1}(\nu(f) \otimes C)=z^{2}$, where $z$ is integral in $X\left(\lambda_{1}(\nu(f) \otimes C)\right.$ obviously is $)$. The proposition follows by factoring out all the powers of 2 possible and then taking square roots.

Proposition (10) actually contains the nonimmersion result, and we use known techniques (Feder [4]) to get it out, reducing the known integrality of $\sqrt{\lambda_{1}(\nu(f) \otimes C)}$ to that of a simpler polynomial. Let $P_{k}(X)=4+T_{k}(X)$, and we write (formally) $T_{k}(X)=y^{k}+y^{-k}+2$ (where $y$ is defined by $y+y^{-1}=$ $X+2)$. The roots of $P_{k}(X)$ and $y^{k}+y_{+2}^{-k}$ are related in a nice way, namely, if $y=y_{0}$ is a root of $y^{k}+y^{-k}+2$, then $x=x_{0}=y_{0}+y_{0}^{-1}-2$ is a root of $P_{k}(X)$. However, the roots of $y^{k}+y^{-k}+2=0$ are rather simple.

Claim. The roots of $y^{k}+y^{-k}+2=0$ are given by

$$
y_{m}=\exp [(2 m-1) \pi i / k], \quad m=0, \ldots, k .
$$

Proof. $y^{k}+y^{-k}+2=y^{-k}\left(y^{k}+1\right)^{2}$. Hence,

$$
X_{m}=\exp \frac{(2 m-1) \pi i}{k}+\exp \frac{2((k-m+1)-1) \pi i}{k}-2, \quad m=1, \ldots, k,
$$

are the zeros of $P_{k}(X)$. Notice that $X_{m}=X_{k-m+1}$, so that all the roots of $P_{k}(X)$ occur with even multiplicity, provided that $m \neq k-m+1$, i.e. $k \neq$ $2 m-1$. Thus, if $k$ is even, $P_{k}(X)$ is the square of an integral polynomial.

Now, write $k=2^{l}(2 m-1), k^{\prime}=2^{l}$. Then, for $f=0,1, \ldots, 2^{l}-1$, the root $X_{m+j(2 m-1)}$ of $P_{k}(X)$ is equal to the root $X_{j+1}^{\prime}$ of $P_{k}^{\prime}(x)$ (this is a trivial computation). This gives

Lemma (11). $P_{k}(X)=P_{k,}(X)(Q(X))^{2}$, where $\operatorname{deg} Q=2^{l}(m-1)$, and $Q(X)$ has constant term 1 . 
Proof. See Feder [4].

We recall the fact that in truncated polynomial rings, polynomials with constant term 1 are invertible (the case we have in mind is $Z[X] /\left(X^{[n / 2]}\right)$ ). This fact together with our analysis and Proposition (10) implies

Lemma (12). With the same conditions as in Proposition (10),

$$
2^{k-n}\left[1 / 4 P_{\beta^{\prime}}(X)\right]^{(k+1) / 2}[1+1 / 4 X]^{-(n+1) / 2} \cdot\left[1 / 4 P_{d^{\prime}}(X)\right]^{1 / 2\left[1 / 4 P_{(e \beta)^{\prime}}(X)\right]^{-1 / 2}}
$$

is an integral polynomial in $X$, where $r^{\prime}=$ largest power of 2 dividing $r$.

Hence the problem is reduced to the case where $\beta, e$ and $d$ are powers of 2. Recall that if $k=2^{r}$, with $r>0, P_{k}(X)$ is a square,

Reducing modulo 2

$$
P_{k}(X)=\left(P_{k}(X)\right)^{2} \text {. }
$$

$$
P_{k}(X) \equiv\left(R_{k}(X)\right)^{2}=R_{k}\left(X^{2}\right)(\bmod 2) .
$$

But, recall

$$
P_{k}(X) \equiv y^{k}+y^{-k} \equiv\left(y+y^{-1}\right)^{k} \equiv X^{k}(\bmod 2)
$$

Thus

$1 / 2 R_{k}(X)=1 / 2 P_{k}(\sqrt{X})=1 / 2 X^{k / 2}+$ integral polynomial with constant term 1.

If $k>n-1$, then $1 / 2 k>[1 / 2 n]$, and because $X^{[n / 2]}=0, R_{k}(X)$ is an integral polynomial with constant term 1 (and hence we can eliminate $P_{k}(X)$ from our integrality condition). If we now let $\alpha(r)=$ the number of 1 's in the dyadic expansion of $r$, we get the main result.

Theorem (13). Suppose $n$ is even, $d$ odd, and $\beta=2^{s}(2 r-1)$ with $2^{s}>$ $n-1$. If $f: V^{n}(d) \rightarrow V^{k}(e)$ is an immersion and $\operatorname{deg}(f)=\beta$, then $k \geq 2 n-$ $\alpha(n-2)-2$.

Proof. The proof breaks down into a number of similar cases; we do one case. Note that since we took $\beta \equiv 0(\bmod 2)$, we must have $d \equiv 1$ in order that $\nu(f)$ be a spin bundle, and so $P_{d^{\prime}}(x)=x+4$. We do the case $k \equiv 0$, $e$ $\equiv 1$. We have

$$
\left.2^{k-n\left(1 / 4 P_{\beta^{\prime}}(X)\right)^{1 / 2(k+1)}(1+1 / 4 X)^{-1 / 2(n+1)} \cdot(1+1 / 4 X)^{1 / 2}(1 / 4 P}(e \beta)^{\prime}(x)\right)^{-1 / 2}
$$

is integral. But, $\beta^{\prime}=2^{s},(e \beta)^{\prime}>2^{s}$ and $2^{s}>n-1$ by assumption; we can eliminate $P_{\beta^{\prime}}$ and $P_{(e \beta)^{\prime}}$ and (10) is integral if and only if

$$
2^{k-n}(1+1 / 4 X)^{-n / 2} \text { is an integral polynomial. }
$$

We look at the coefficient of the highest nonvanishing power, $X^{(n-2) / 2}$, in the expansion of (11). This coefficient is $K=2^{k-n} 2^{-n+2} 2^{2 a}(n-2) / 2-$ 
$a(n-2)$. odd number. In order that this be an integer, we need $(k-n)-$ $n+2+2 \alpha\left(\begin{array}{c}n-2 \\ 2\end{array}\right)-\alpha(n-2) \geq 0$, but $n-2$ is even, so $\alpha\left(\left(_{2}^{n-2}\right)=\alpha(n-2)\right.$; thus we get

$$
k-2 n+a(n-2)+2 \geq 0, \quad \text { or } \quad k \geq 2 n-a(n-2)-2 .
$$

The other cases are similar.

Note. Feder [4] carried out this analysis for $C P^{n}=V^{n+1}(1)$ in $C P^{k}=$ $V^{k+1}(1)$, and our result reduces to his if $d=e=1$. Now, if we let $\beta=0$ in Proposition (12) we get a result about immersions in $R^{n}$ :

Theorem (14). If $V^{n}(d)$ immerses in $S^{k-1}, d$ odd, then $k \geq 2 n-a(n-2)$ -2 .

Proof. An argument similar to the one in Theorem (13).

Finally, if we combine Proposition (1) of $\$$ II with Theorem (13), we get

Theorem (15). Let $n$ be even, $d$ odd, $\beta=2^{r}(2 s-1), 2^{r}>n-1$.

(1) If $n-2$ is not a power of 2, suppose e does not divide $\beta^{n-1} d$ or $k<2 n-\alpha(n-2)-2$.

(2) If $n-2$ is a power of 2, suppose $k<2 n-3$.

Then there exists no immersion $f: V^{n}(d) \rightarrow V^{k}(e)$ with $\operatorname{def}(f)=\beta$.

\section{REFERENCES}

1. J. F. Adams, Vector fields on spheres, Ann. of Math. (2) 75 (1962), 603-632. MR $25 \# 2614$.

2. A. Andreotti and T. Frankel, The Lefschetz theorem on hyperplane sections, Ann. of Math. (2) 69 (1959), 713-717.

3. E. Dyer, Cohomology theories, Math. Lecture Note Series, Benjamin, New York, 1969. MR 42 \#3780.

4. S. Feder, Immersions and embeddings in complex projective spaces, Topology (4) (1965), 143-158. MR 32 \#1717.

5. F. Hirzebruch, Neue topologische Methoden in der algebraischen Geometrie, Ergebnisse der Mathematik und ihrer Grenzgebiete, Heft 9, Springer-Verlag, Berlin, 1956; English transl., Die Grundlehren der math. Wissenschaften, Band 131, SpringerVerlag, New York, 1966. MR 18, 509; 34 \#2573.

6. D. Husemoller, Fiber bundles, McGraw-Hill, New York, 1966. MR 37 \#4821.

7. I. M. James. Euclidean models of projective spaces, Bull. London Math. Soc. 3 (1971), 257-276.

8. K. H. Mayer, Eliptische Differentialoperatoren und Ganzzahligkeitssätze für characteristische Zahlen, Topology 4 (1965), 295-313. MR 33 \#6650.

9. J. Milnor, Representation rings of some classical groups, Mimeographed notes, Princeton, 1958.

10. R. Thom, Quelques propriétes globales des variétés différentiables, Comment. Math. Helv. 28 (1954), 17-86. MR 15, 890.

DEPARTMENT OF MATHEMATICS, UNIVERSITY OF BRITISH COLUMBIA, VANCOUVER (8), BRITISH COLUMBIA, CANADA

Current address: Department of Mathematics, University of Washington, Seattle, Washington 98105 